\title{
Optimal focal length of primary mirrors in Fresnel linear collectors
}

\author{
Paola Boito ${ }^{\mathrm{a}, \mathrm{b}}$, Roberto Grena ${ }^{\mathrm{c}, *}$ \\ ${ }^{a} X L I M-D M I$, Université de Limoges UMR CNRS 7252, 123 av. A. Thomas, 87060 \\ Limoges, France \\ ${ }^{b}$ CNRS, Université de Lyon, Laboratoire LIP (CNRS, ENS Lyon, Inria, UCBL, 46 allée \\ d'Italie, 69364 Lyon Cedex 07, France \\ ${ }^{c}$ C. R. ENEA Casaccia, via Anguillarese 301, 00123 Roma, Italy
}

\begin{abstract}
In a linear Fresnel plant adopting slightly curved primary mirrors (cylindrical or parabolic), a significant gain in the radiation collection can be achieved using primary mirrors with different focal lengths, dependent on the position of the mirror with respect to the receiver. This work introduces a universal function that gives the optimal focal length of a mirror, given only its position relative to the receiver and the latitude, for a NS-oriented collector with a flat horizontal effective target. In a solar plant with the focal lengths defined by this function, the efficiency gain with respect to a solar field adopting identical mirrors is estimated in the range $1.5-6 \%$, with the gain increasing if mirror imperfections or tracking errors are present: this means that the regulation of the focal lengths is especially useful in containing the loss of efficiency due to defects. The given rule is tolerant to errors in the focal regulation (up to 10\%). The function can be used as a reference for future projects, or as starting point for more refined optimizations.
\end{abstract}

Keywords: Keyword1, Keyword2, Keyword3

PACS: PACSN1, PACSN2

\section{Introduction}

In Linear Fresnel concentrators (LFC in the following), a linear fixed receiver is suspended above a solar field composed of strips of mirrors (Di Canio et al., 1979; Feuermann, 1991; Francia, 1968; Montes et al., 2014; Zhu et al., 2014). The mirrors are usually slightly concentrating, and each strip rotates on a fixed horizontal axis in order to reflect the sun radiation towards the receiver. LFCs

\footnotetext{
* Corresponding author.

Email addresses: paola.boito@unilim.fr (Paola Boito), roberto.grena@enea.it (Roberto Grena)
}

Preprint submitted to Elsevier

April 21, 2017 
received a widespread attention in recent years, because they have many advantages with respect to more traditional single-mirror concentrators: the fixed receiver, the larger collection area for each receiver, the smaller moving parts and the lower cost of the optical components. A large number of studies can be found in the literature discussing LFC configurations (Abbas et al., 2013; Grena and Tarquini, 2011; Häberle et al., 2002; Mills and Morrison, 2000; Zhu and Huang, 2014) or comparing LFCs with linear troughs (Giostri et al., 2013; Morin et al., 2012; Schenk et al., 2014). Many full scale prototypes have already been built (Areva, 2015; Bernhard et al., 2008, 2009; Novatec, 2015; Solar Power Group, 2015).

Even neglecting the measurements and specifications of the receiver, the geometry of an LFC is defined by a large number of parameters, which can be changed independently, at least in principle: the widths, positions and focal lengths of each mirror are all independent variables, therefore the solar field geometry depends on $3 N_{m}$ degrees of freedom, where $N_{m}$ is the number of mirrors. Optimization techniques were theoretically investigated and discussed in the literature. Some authors have reduced the degrees of freedom by means of simple criteria, such as the absence of shadowing up to a certain incidence angle (Nixon and Davies, 2012) or other theoretical criteria (Abbas and MartinesVal, 2015; Chaves and Collares-Pereira, 2010). The reduction of the number of parameters is not strictly required: in Boito and Grena (2016) a method to perform a full optimization of all the geometric parameters of an LFC was presented, assuming that the dependence of the plant cost from the parameters is given. This method optimizes the ratio between the year-long collected radiation and the plant cost. Assuming a plausible cost model, it was shown that a full optimization produced a relative gain of $12 \%$ w.r.t. a uniform, adjacent-mirrors configuration, and of almost $5 \%$ w.r.t. a uniform configuration with 3 optimized parameters (mirror width, mirror focal, spacing between mirrors). The paper also presented the results of partial optimizations: these were performed by enforcing uniformity constraints (e.g., uniform spacing, or uniform width) on some - but not all - of the parameters, in order to highlight the effect of changes on each parameter.

The method proposed in Boito and Grena (2016) cannot be used to assess universal criteria for LFC design, since its results are dependent on the price model of the plant. But a result obtained in one of the partial optimizations presented in Boito and Grena (2016) suggested a possible universal rule. When optimizing the focal lengths of the mirrors, under the hypothesis of equal width and of uniform spacing, a significant gain was obtained w.r.t. a simple optimization with equal focal lengths, more than the $60 \%$ of the gain obtained with a full optimization. Note that the chosen price model did not include the focal lengths: indeed, it was assumed that the curvature did not affect the mirror cost, a reasonable assumption for small curvatures. Therefore, the gain was only due to the increased optical collection. Moreover, the shadowing and blocking effects were small, because the mirrors were well-spaced, following the result of the previous optimization. So it is likely that the optimal focal length of a mirror is essentially linked to the properties of the mirror itself (particularly its 
position relative to the receiver), and it does not depend much on the global structure of the plant.

This remark suggests the existence of a universal function that, given the geometry of a mirror relative to the receiver, computes the focal length that maximizes the year-long optical collection. Of course, general properties of the plant structure, such as orientation and latitude, also need to be taken into account. Such a function does not depend on any price parameter, so it gives useful criteria for designing solar fields, even when price parameters are not known (e.g., in the case of innovative plants).

One may reasonably think that the optimal focal length of a mirror should equal the distance from the receiver. However, in Boito and Grena (2016) it was shown that this is not a good criterion: if in the optimal configuration the computed focal lengths were substituted by the distances from the receiver, the efficiency gain completely disappeared. This is due to the fact that a Fresnel mirror almost never works in-focus, and the regulation of the focal assuming an in-focus concentration is useless. Optimization of the focal lengths is a more subtle issue that requires specific computations.

In this work we perform such computations on a plant with NS orientation and a flat, horizontal receiver. Both the case of cylindrical and of parabolic mirrors are studied. First, it is assessed which of the geometric parameters of the mirrors are really useful for defining an optimal focal length, with the aim of reducing the number of parameters as much as possible. We anticipate that, allowing a small tolerance $(1.2 \%)$ on the collected radiation w.r.t. the optimal value, the only relevant parameters that determine the optical focal lengths are the position of the mirror relative to the receiver, and the latitude of the plant. Neither the width of the mirror nor the width of the receiver affects significantly the results, for any plausible configurations.

This means that it would be possible to define a simple function that determines the optimal focal length of the mirror, given only the latitude and the position of the mirror w.r.t. the receiver. Such a function is numerically computed and the results are fitted to give a very simple, analytic form of the function, valid both for parabolic and cylindrical mirrors. Tests on some plant configurations are then performed, with a full optical simulation, in order to compute the efficiency gain that would be obtained when applying this design criterion.

\section{Model and computations}

In the computations, a single mirror is considered: no shadowing and blocking from adjacent mirrors are taken into account. The mirror is NS-oriented. The distance between its rotation axis and the center of the solar field (the point directly below the center of the receiver) will be called $x$, its semi-width will be called $w$ and its focal length will be called $f$. Both cylindrical and parabolic mirrors are considered. The receiver is flat and horizontal; its semi-width will be denoted as $l$. The shadow projected from the receiver on the mirror is neglected too. The height of the receiver above the mirror axes is fixed to 1 , since the 
system is scale invariant; i.e., all the given values of $x, w, s$ and $l$ should be read as ratios w.r.t. the height of the receiver (e.g., for a $10 \mathrm{~m}$ high receiver, a value $w=0.05$ corresponds to a $1 \mathrm{~m}$ wide mirror). The scheme of the system considered in the computations is shown in Figure 1.

>> > approx. position of Figure $1<<<$

The quantity optimized in order to find the ideal focal length is the average geometrical collection over a year, i.e., all the reflectivities are considered to be 1. This is computed according to the scheme presented in Boito and Grena (2016), which we quickly recall:

- The average efficiency is computed integrating the pointwise efficiency (i.e., the efficiency given the sun position) over the distribution of the sun position during the year. Corrections due to the variations of the distance Earth-Sun and to the air mass are included. The sky is assumed to be "averagely clear", i.e., meteorological conditions are supposed to affect uniformly the radiation distribution. The integration variables are the declination $\delta$ and the hour angle $H$ of the sun, and the integration methods are Gauss-Lobatto over $H$ and Gauss-Chebyshev on $\delta$.

- The pointwise efficiency is computed integrating on the sun profile the efficiency for collimated rays; the sun profile is considered to be Lambertian.

- The efficiency for collimated rays is computed by calculating with high precision the fraction of the mirror that projects the reflected rays within the receiver opening.

The parameters that can, in principle, affect the value of the optimal focal length are the latitude of the plant, the position of the mirror, the width of the receiver and the width of the mirror. All of them have been studied in order to assess their effects on the optimal focal length, considering all the plausible configurations. A configuration is considered plausible if:

- $0.01 \leq w \leq 0.1$

- $0.005 \leq l \leq 0.05$;

- given $w$ and $l$, the efficiency for the optimal focal length should be at least $80 \%$ of the radiation collected by the mirror.

The first two conditions allow all the reasonable receiver and mirror sizes for a Fresnel plant: e.g., if the receiver is $10 \mathrm{~m}$ high, the receiver can be from 10 $\mathrm{cm}$ to $1 \mathrm{~m}$ wide, and the mirrors can go from $20 \mathrm{~cm}$ to $2 \mathrm{~m}$.

The last condition allows us to discard mirrors that are clearly oversized w.r.t. the receiver. An ideally-sized mirror would have the minimum width required to send all the radiation in the receiver opening; a larger mirror is a 
useless waste of materials and space. However, this condition can be relaxed due to building constraints (e.g., required uniformity) or for error tolerance. So, an oversizing of the mirror up to a (purely geometrical) $20 \%$ of radiation loss is allowed.

Of course, the use of an undersized mirror is undesirable too; but in this analysis no conditions were introduced to avoid undersizing, since it gives no problems in the computation of optimal focal lengths. For a strongly undersized mirror, a large set of focals are equally optimal, giving the maximum possible collection.

The main goal of the present work is to give a focal length function that is as universal and simple as possible, eliminating all the parameters that are not strongly significant. The starting point is a function that, given the parameters $x, \phi, w$ and $l$, outputs the optimal focal length. A range of acceptable almost-optimal focals has been computed, where "almost optimal" means that the efficiency should be within $1.2 \%$ of the true optimal efficiency. Now, suppose that a parameter (say, $w$ ) varies across its range. If the various focal ranges computed for the different values of $w$ contain a common value, then this common value can be assumed as an almost-optimal focal length for all the mirror widths and the parameter $w$ can be eliminated, thus defining a new function that gives a focal length with efficiency within $1.2 \%^{1}$ of the true optimal efficiency and is not dependent on the mirror width. A similar argument applies to all the parameters.

Using this approach, it turns out that $w$ and $l$ can be eliminated from the list of significant parameters. This fact can be easily understood imagining the limit case of a very small mirror and collector: in such a limit, the only relevant parameters can be $x$ and $\phi$. A Fresnel plant can be considered a feasible approximation of this limit because the aim of a Fresnel configuration is to build primary mirrors that are very small w.r.t. the total collecting area, and to concentrate the radiation towards a line. So it is reasonable that the only relevant parameters would be the same. Such conclusions are verified by computation, within the given tolerance.

The function that gives the optimal focal length is fitted w.r.t. $x$ and $\phi$ in the simplest possible form. Note that $\phi$ and $x$ play different roles here: $\phi$ is fixed once the plant location is given, at which point only the variable $x$ matters for the computation of focal length and for the design of the plant. It is therefore preferable to see $\phi$ as an external parameter; once $\phi$ has been fixed, one is left with a function of $x$ only. In practice, for each of the latitude angles under consideration, a power-law fit was applied with respect to $x$, followed by an estimation of the dependence of the fit parameters on $\phi$. The function that gives the optimal focal vs $x$, given the latitude $\phi$, will be denoted as $F_{\phi}(x)$.

\footnotetext{
${ }^{1}$ The $1.2 \%$ threshold is, in fact, an a posteriori choice, since it is the minimum tolerance for which both $w$ and $l$ can be eliminated.
} 


\section{Results}

As pointed out earlier, a tolerance of $1.2 \%$ on the optimal collection is enough to obtain a common focal length for $x$ and $\phi$, eliminating $w$ and $l$ from the relevant parameters. This fact was accurately checked by computing, for all the plausible combinations of $w$ and $l$, the ranges of almost-optimal focal lengths (within the prescribed tolerance) and showing that the ranges included common values. More than 2000 configurations of $w$ and $l$ were tested for each pair $(x, \phi)$, selecting the plausible configurations and then computing the intersection of all the ranges found, in order to determine, for $(x, \phi)$, the range of focals which are almost-optimal for all the values of $w$ and $l$. Figure 2 shows the ranges obtained vs the $x$ variable, for the two extreme values of $\phi$ considered in the computations ( 0 and $60 \mathrm{deg}$ ). Results for parabolic and cylindrical mirrors are essentially the same, suggesting that at such small curvatures the two shapes are indistinguishable.

As mentioned above, a fit with respect to the variable $x$ was performed for each value of $\phi$. A good fit of $F_{\phi}(x)$ is given, for all the latitudes, by the simple power law

$$
F_{\phi}(x)=a_{\phi}+b_{\phi} x^{1.6} .
$$

The exponent of $x$ was initially considered a fit parameter depending on $\phi$, but results showed that the common value 1.6 gave equally good results.

Note that this is not a classical fit problem where a single experimental result is given for each value of $x$ and the fitted curve should minimize the distance from the experimental points. Here, each $x$ is associated with a whole range of values for $F_{\phi}(x)$, and the fitted curve should stay inside these intervals. For each $\phi$, there are many values of the pair $\left(a_{\phi}, b_{\phi}\right)$ that satisfy this requirement, and it makes sense to choose a pair that is "in the middle" of this admissible set, so that the quality of the fit is not too sensitive to perturbations. The approach adopted here involves determining experimentally the admissible set in the $(a, b)$ plane and then computing its barycenter, together with an error estimate $\left(\sigma_{a}, \sigma_{b}\right)$ given by the standard deviation. Two of the fit functions, for latitudes 0 and $60 \mathrm{deg}$, are shown in Figure 2.

$>>$ approx. position of Figure $2<<<$

The next step consists in estimating the dependence of $a_{\phi}$ and $b_{\phi}$ on $\phi$, that is, fitting $a_{\phi}$ and $b_{\phi}$ as functions of $\phi$. For each $\phi$ the fitting functions for $a_{\phi}$ and $b_{\phi}$ should be as close as possible to the barycentric values found earlier, and stay in the intervals $\left[a_{\phi}-\sigma_{a}, a_{\phi}+\sigma_{a}\right]$ and $\left[b_{\phi}-\sigma_{b}, b_{\phi}+\sigma_{b}\right]$, respectively.

It turns out that both $a_{\phi}$ and $b_{\phi}$ have a quadratic dependence on $\phi$ :

$$
a_{\phi}=1.0628+0.0467 \phi^{2}, \quad b_{\phi}=0.7448+0.1394 \phi^{2} .
$$

In (2), the latitude must be given in radians. The fit curves are shown in Figure 3 .

>> > approx. position of Figure $3<<<$ 
So, the main result of this work can be summarized as follows:

Given a LFC with NS orientation, at a latitude $\phi$, with a flat horizontal effective opening of the receiver, adopting parabolic or cylindrical mirrors, the focal lengths of the mirrors should be chosen according to the law

$$
F_{\phi}(x)=a_{\phi}+b_{\phi} x^{1.6}
$$

where $x$ is the ratio between the abscissa of the mirror (in absolute value) and the height of the receiver. The coefficients $a_{\phi}$ and $b_{\phi}$ are given by ( $\phi$ in radians):

$$
a_{\phi}=1.0628+0.0467 \phi^{2}, \quad b_{\phi}=0.7448+0.1394 \phi^{2} .
$$

No simple physical interpretations of the formulas have been found. More physically sound fit functions were tried, to no avail: dependence on $x$ related to the distance mirror-receiver, or to the theoretical curvature of a true Fresnel reflector, or trigonometric dependence on $\phi$, gave no satisfying results, while the presented simple power laws did. In particular, the ratio optimal focal / distance mirror-receiver is not constant, but it increases significantly with $x$; this means that an optimization assuming a constant ratio (Morin et al., 2006) may not always be the best option. The difficulties in finding physically meaningful relations is probably due to the fact that the efficiency is produced by many different effects (average on the sun position, air mass effect, mirrors working not-in-focus) and simple physical relations cannot be extracted. However, it is remarkable that simple, universal power laws can be found anyway, with a limited number of fit parameters.

\section{Test of the formula}

The law to find the optimal focal length of the mirrors of the solar field $(1,2)$ was tested on a typical plant configuration, already optimized for 3 parameters (mirror width, mirror spacing, mirror common focal length). The configuration used for comparison is the configuration called UC in Boito and Grena (2016). The comparison was made by means of full optical simulations, including shadowing, blocking, shadow from the receiver, and mirror slope errors. The gain obtained applying the given formula to the focals, w.r.t. the original configuration, was computed, with special attention to the effect of defects and errors: since all the previous computations were made for a perfect system, their usefulness in a real system with error imperfections, tracking errors and limited accuracy on the mirror curvature is not guaranteed. Three types of errors are considered: mirror imperfections, overall tracking errors and errors on the focal lengths.

Figure 4 shows the efficiency gain, in percent, plotted w.r.t. the standard deviation of slope errors of the mirrors, assumed Gaussian and isotropic. From the figure, one can see that the gain is not very large (about 1.5\%) for perfect mirrors, since the system was already optimized; but it increases significantly when mirror imperfections are taken into account. This means that regulating 
the focal lengths of the mirrors according to the given formula is especially useful in containing the loss due to mirror imperfections. For a typical value of slope deviation (0.2 degrees) the gain obtained is more than $3 \%$. For lower quality mirrors (slope deviation around $0.4 \mathrm{deg}$ ) the gain is almost $6 \%$.

Figure 4 also shows the efficiency gain for different tracking errors. The tracking error is assumed to be coherent, i.e., all the mirrors have the same deviation from perfect alignment. This is a limiting case, not very likely to occur in practice: it can only happen if unsuitable tracking algorithms are used. Moreover, the behaviour of the curve is quite extreme: the gain can be very large (more than $25 \%$ ) for a tracking error around $0.6 \mathrm{deg}$, then it falls abruptly.

$>>>$ approx. position of Figure $4<<<$

Figure 5 shows how the efficiency gain is tolerant for errors on the focal length itself. This is an especially important point, since regulating the focal length with accuracy can be difficult for small curvatures, with the constraint of keeping the cost as low as possible. An error of $1 \mathrm{~mm}$ on the edge height relative to the center of the mirror, for example, can give an error around $0.5 \mathrm{~m}$ in the focal length for a mirror $1 \mathrm{~m}$ wide with a focal around $10 \mathrm{~m}$. So, a test was performed assuming that each mirror focal has a random Gaussian error with standard deviation corresponding to a certain percentage of the nominal focal (the efficiency was computed averaging on 50 configurations). The figure shows that errors on the focals do not cancel the advantage of the focal regulation according to $(1,2)$. On the contrary, they seem to increase the effect: even if the curve does not appear smooth (probably an effect of the sample for average), an increasing trend is clearly recognizable. So, the regulation of focals is also useful in containing the effect of errors on the focals themselves.

>> approx. position of Figure $5<<<$

\section{Conclusions}

A universal formula for almost-optimal focal lengths of the primary mirrors in a NS-oriented LFCs was found, assuming a horizontal effective opening of the receiver. The formula depends only on the relative position mirror-receiver, and on the latitude of the plant. When the focal lengths of an already optimized, uniform focal LFR are changed according to the formula, an efficiency gain of the order of some percent is obtained, and the regulation is especially useful in containing the effect of slope deviations: for low-quality mirrors, the gain can be almost $6 \%$. The focal regulation itself is tolerant to errors and the efficiency gain does not disappear (in fact, it increases) for all the tested configurations, that is, up to a tolerance of $\pm 10 \%$ on the focal length. So, the formula can be useful in practice. Since the formula optimizes only the optical collection and does not require any cost parameters, it can be applied to any LFC plant with NS orientation and a horizontal effective opening of the receiver, even if cost 
parameters are unknown. The formula can be employed in the design of new plants or used as the starting point of more refined optimization, including more variables and cost parameters.

Abbas, R., Munoz-Anton, J., Valdes, M., Martinez-Val, J.M., 2013. High concentration linear Fresnel reflectors. Energy Conv. Manag. 72, 60-68.

Abbas, R., Martnez-Val, J.M., 2015. Analytic optical design of linear Fresnel collectors with variable widths and shifts of mirrors. Renew. Energy 75, 81-92.

Areva, online (oct. 2016) at URL: http://www.areva.com/EN/global-offer725/concentrated-solar-power-renewable-energies-solutions.html.

Bernhard, R., Laabs, H.J., de Lalaing, J., Eck, M., Eickhoff, M., Pottler, K., Morin, G., Heimsath, A., Georg, A., Haberle, A., 2008. Linear Fresnel collector demonstration on the PSA. Part I - Design, construction and quality control. 14th International SolarPACES Conference, Las Vegas, USA.

Bernhard, R., Lalaing, J., Kistner, R., Eck, M., Eickhoff, M., Feldhoff, J.F., Heimsath, A., Hulsey, H., Morin, G., 2009. Linear Fresnel Collector demonstration at the PSA - operation and investigation. 15th International SolarPACES Conference, Berlin, Germany.

Boito, P., Grena, R., 2016. Optimization of the geometry of Fresnel linear collectors. Sol. Energy 135, 479-486.

Chaves, J., Collares-Pereira, M., 2010. Etendue-matched two-stage concentrators with multiple receivers. Sol. Energy 84, 196-207.

Di Canio, D.G., Tretyl, W.J, Jur, F.A., Watson, C.D., 1979. Line-focus Solar Thermal Central Receiver Research Study. FMC Corporation, Santa Clara, CA, Final Report 1977-79, DOE/ET/20426-1.

Feuermann, D., Gordon, J.M., 1991. Analysis of a two-stage linear Fresnel reflector solar concentrator. J. Sol. Energy Eng. Trans.-ASME 113, 272279.

Francia, G., 1968. Pilot plants of solar steam generating stations. Sol. Energy $12,51-64$.

Giostri, A., Binotti, M., Silva, P., Macchi, E., Manzolini, G., 2013. Comparison of Two Linear Collectors in Solar Thermal Plants: Parabolic Trough Versus Fresnel. J. Sol. Energy Eng. Trans.-ASME 135, 011001.

Grena, R., Tarquini, P., 2011. Solar linear Fresnel collector using molten nitrates as heat transfer fluid. Energy 36, 1048-1056.

Häberle, A., Zahler, C., Lerchenmüller, H., Mertins, M., Wittwer, W., Trieb, F., Dersch, J., 2002. The Solarmundo Line Focusing Fresnel Collector: Optical Performance and Cost. 11th SolarPACES Conference, Zurich, Switzerland. 
Mills, D.R., Morrison, G.L., 2000. Compact linear Fresnel reflector solar thermal powerplants. Sol. Energy 68, 263-283.

Montes, M.J., Rubbia, C., Abbas, R., Martinez-Val, J.M., 2014. A comparative analysis of configurations of linear Fresnel collectors for concentrating solar power. Energy 73, 192-203.

Morin, G., Dersch, J., Platzer, W., Eck, M., Haberle, A., 2012. Comparison of Linear Fresnel and Parabolic Trough Collector power plants. Sol. Energy 86, $1-12$.

Morin, G., Platzer, W., Eck, M., Uhlig, R., Hberle, A., Berger, M., Zarza, E., 2006. Road map towards the demonstration of a linear Fresnel collector using single tube receiver. 13th International Symposium on Concentrated Solar Power and Chemical Energy Technologies, Seville, Spain.

Nixon, J., Davies, P., 2012. Cost-exergy optimisation of linear Fresnel reflectors. Sol. Energy 86, 147-156.

Novatec Solar, online (oct. 2016) at URL: http://www.novatecsolar. com/.

Schenk, H., Hirsch, T., Feldhoff, J.F., Wittmann, M., 2014. Energetic Comparison of Linear Fresnel and Parabolic Trough Collector Systems. J. Sol. Energy Eng. Trans.-ASME 136, 041015.

Solar Power Group, online (oct. 2016) at URL: http://www.solarpowergroup. com.

Zhu, J., Huang, H., 2014. Design and thermal performances of Semi-Parabolic Linear Fresnel Reflector solar concentration collector. Energy Conv. Manag. 77, 733-737.

Zhu, G.D., Wendelin, T., Wagner, M.J., Kutscher, C., 2014. History, current state, and future of linear Fresnel concentrating solar collectors. Sol. Energy 103, 639-652. 


\section{Figure captions}

Figure 1: Geometry of the system considered for the computation of the optimal focal length.

Figure 2: Ranges of almost-optimal focal lengths, for different latitudes.

Figure 3: Dependence on the latitude of the parameters $a_{\phi}$ and $b_{\phi}$.

Figure 4: Efficiency gain w.r.t. a typical plant configuration in dependence of slope errors and coherent tracking errors.

Figure 6: Efficiency gain w.r.t. a typical plant configuration in dependence of the relative error in regulating the focal lengths. 